\title{
Pilot Results from the First HIV/AIDS Intervention Among Transgender Women in the Middle East: Gender Affirmation and Social Support from Within Trans Communities in Beirut, Lebanon
}

\author{
Rachel L. Kaplan,, Cynthia El Khoury, ${ }^{2}$ Sara Wehbe, ${ }^{2}$ Norma Lize, ${ }^{2}$ and Jacques Mokhbat ${ }^{3}$
}

\begin{abstract}
Transgender women are 49 times more likely to become HIV positive than all adults worldwide. Data were unavailable for trans women's sexual and mental health in the Middle East and North Africa until 2015-2016, when HIV prevalence (10\%) and suicide attempt (46\%) rates were established in Lebanon. Because of the lack of evidence-based interventions for this marginalized group, the purpose of this study was to pilot test an adapted intervention, "Baynetna," to assess preliminary impact on mental and sexual health. Using the gender affirmative model and community connectedness and social cohesion constructs, we pilot tested Baynetna among sixteen trans women, who attended 6 weekly 3-h trans-facilitated group-delivered sessions in Beirut with post-test assessments at 1, 3, and 6 months. Each participant acted as her own control; we used paired $t$-tests to assess changes at follow-up to evaluate potential differences in outcomes. Positive directionality in intervention impact on gender affirmation satisfaction, community connectedness, and social cohesion was demonstrated. An increase in HIV testing proportion between 6 months before and after enrollment was achieved. Higher social cohesion was associated with less suicidal thoughts $(p=.05)$. There was a significant $(p=.019)$ relationship between more community connectedness and lower depression scores. War event exposure was associated with higher anxiety $(p=.02 ; p=.004)$. Those who reported never having had a sexually transmitted infection had higher gender affirmation satisfaction scores $(7.5,95 \%$ confidence interval: 1.4-13.6) $(p=.019)$. Increases in community connectedness, social cohesion, and gender affirmation satisfaction scores confirm Baynetna's proposed mechanisms of impact. Proportional HIV testing increases demonstrate prevention objectives' progress. The significant results contribute to confidence in Baynetna's mechanisms of action and support the hypothesis that the Gender Affirmation model is applicable to the Lebanese context. We have shown for the first time that the adapted intervention, and its constructs, are applicable in this context outside the United States.
\end{abstract}

Keywords: transgender women, Lebanon, Middle East and North Africa (MENA), community, mental health, social support

\section{Introduction}

T RANSGENDER WOMEN (hereafter "trans women," which includes individuals whose identities are not women/are trans feminine ${ }^{1}$ ) are 49 times more likely to become HIV positive than all adults of reproductive age worldwide. ${ }^{2}$ Global efforts to address the HIV epidemic emphasize improving access to HIV testing, particularly among key populations. ${ }^{3}$ HIV testing barriers that trans women experience include stigma, discrimination, and marginalization. ${ }^{4,5}$ Members of this population in Lebanon experience these barriers due in part to rigid expectations of

\footnotetext{
${ }^{1}$ Department of Obstetrics, Gynecology, and Reproductive Sciences, University of California, San Francisco (UCSF), San Francisco, California, USA.

${ }^{2}$ Independent Researcher, Beirut, Lebanon.

${ }^{3}$ School of Medicine, Lebanese American University, Beirut, Lebanon.

(C) Rachel L. Kaplan et al. 2020; Published by Mary Ann Liebert, Inc. This Open Access article is distributed under the terms of the Creative Commons Attribution Noncommercial License (http://creativecommons.org/licenses/by-nc/4.0/) which permits any noncommercial use, distribution, and reproduction in any medium, provided the original author(s) and the source are cited.
} 
binary gender expression, ${ }^{6}$ which can hinder HIV testing, early detection, and linkage to care.

Despite the staggering health disparities in HIV risk among trans women in other regions globally, HIV testing rates among trans women in the Middle East and North Africa (MENA) are mostly unknown. MENA is one of the only regions where new infections are still on the rise. ${ }^{7}$ Furthermore, countries in MENA are far from the objective of attaining the UNAIDS 90-90-90 goals by $2020 .^{8}$ The UNAIDS country report for Lebanon estimates a steady increase in the number of new infections of HIV as well as in the number of people living with HIV, ${ }^{9}$ documenting the urgency of improving HIV testing among trans women.

No data were available for trans women's sexual health in MENA until results from a cross-sectional study in Lebanon were published in 2015 and 2016. ${ }^{6,10,11}$ Because of societal and contextual factors in addition to structural barriers that limit opportunities, many trans women engage in survival sex work. ${ }^{6}$ We now know that the documented prevalence of HIV among trans women in Lebanon is $10 \%$, which is likely an underestimate, ${ }^{10}$ and that attempted suicide is incredibly high at $46 \%$. $^{11}$ The HIV prevalence rate among trans women is higher than another key population within the country for which data are available; among men who have sex with men, HIV prevalence is $1.5 \%-3.6 \% .^{12,13}$ High rates of condomless receptive anal intercourse $(57 \%)$ and never having been tested for HIV (40\%) characterize trans women in this low prevalence setting ${ }^{10}$ and demonstrate the need for effective interventions to reach the 90-90-90 goals ${ }^{8}$ in Lebanon.

Globally, trans women also experience disparate rates of poor mental health, such as depression $(42 \%-64 \%)^{14-16}$ and suicide attempts $(31 \%-41 \%){ }^{15,17,18}$ In Lebanon, the prevalence rate of reported suicide attempts among trans women is much higher $(46 \%)$ than among the general population $(2 \%) .{ }^{19}$ Of trans women reporting past suicide attempt, only one of 53 participants had received some kind of formal mental health services. ${ }^{11}$

Despite such high risk for HIV and poor mental health sequelae, there are currently no evidence-based interventions to address sexual and mental health for this marginalized group. Effective culturally relevant prevention strategies are urgently needed for trans women. In Lebanon, HIV testing is available at nongovernmental organizations (NGOs), government hospitals, and private laboratories. Trans-inclusive services are available (e.g., as members of the broader lesbian, gay, bisexual, transgender, queer, plus (LGBTQ+) community and/or trans women who are refugees), but services and programming specifically for or led by trans women are almost nonexistent.

To begin to address low HIV testing rates and high HIV risk, we adapted an existing intervention (TransAction) that was developed in Los Angeles, California for trans women to the Lebanese context. TransAction, one of the few existing theory-based HIV prevention interventions for trans women with mixed HIV status, has been operating since its first implementation in 1995. It is a trans-facilitated multisession group-level intervention that addresses the unifying lived experiences of trans women who are at elevated risk of acquiring or transmitting HIV and has demonstrated success in decreasing sexual risk. ${ }^{20}$

The ADAPT-ITT Model ${ }^{21}$ was used to adapt TransAction culturally to the Middle Eastern environment using in-depth interviews, focus group discussions, and community advisory board meetings from April to October 2016. The adaptation process has resulted in an Arabic/English glossary of relevant terms, Arabic and English intervention manuals, and successful enrollment of sixteen participants who engaged in a pilot test to determine feasibility, acceptability, and preliminary effects. "Baynetna," Arabic for "between us" and a play on the word for "girls," is the intervention's adapted name and consists of six trans-facilitated group-delivered sessions that seek to improve mental health, sexual health, and social support.

The model of gender affirmation $(\mathrm{GA})^{22}$ guided study design and development. It is a trans-specific model created to examine the role of gender affirmation in risk-taking, self-care, and health care-seeking behavior. Preliminary support for the GA model demonstrates how meeting trans women's needs for gender affirmation may decrease risk behavior and increase self-care. Applied to the Lebanese context, this approach would suggest that meeting trans women's needs, such as familial support from other trans women in light of rejection from family of origin, could decrease sexual risk taking and increase self-care, including HIV testing. Baynetna seeks to impact gender affirmation needs and thereby care for oneself.

The concepts of "social cohesion" and "community connectedness" guided this work. Social cohesion is a social/environmental construct that can be described as the perception of mutual aid, trust, and support ${ }^{23}$ and is a construct upon which TransAction was built. ${ }^{20}$ Interventions that seek to create, improve, or foster social cohesion are particularly important for marginalized groups that experience discrimination and exclusion from public life. ${ }^{24}$ Similarly, community connectedness refers to feeling part of a "community of others" that allows individuals to make positive comparisons to other people like them, rather than making negative comparisons based on, in this case, transphobia. ${ }^{24}$ Therefore, among trans women, being connected to and socially cohesive with others in the trans community may play an ameliorative role in the relationship between stigma/discrimination and mental health. ${ }^{24,25}$

Evidence from the United States and Australia suggest that good mental health, resilience, and self-esteem among trans individuals is associated with connectedness to a community of "similar others." 25-29 In fact, connectedness with other trans people during the early stages of identity development has been demonstrated to predict decreases in anxiety and suicide ideation significantly. ${ }^{30}$ Previous qualitative research indicated a lack of trust among trans women in Lebanon. ${ }^{6}$

To address the unique health disparities of trans women by improving trans-specific social support, the purpose of this study was to adapt TransAction to the local context and then to pilot test Baynetna to assess small-scale feasibility, acceptability, and preliminary impact of participation on mental and sexual health. We were also interested in determining whether the Gender Affirmation model proves applicable in a setting outside the United States. In this study, we present the main results and relevant associations of hypothesized mechanisms of action in the following overall domains: gender affirmation; social support (from within trans communities); and mental and sexual health.

\section{Materials and Methods}

A complete description of materials and methods of this study can be found in a previous publication describing the feasibility and acceptability of Baynetna ${ }^{31}$; in this study we briefly outline the methods for ease and accessibility. 


\section{Participants and recruitment}

The pilot test treatment-only convenience sample included 16 trans women who met the eligibility criteria of being 18 years or older, being able to give informed consent in Arabic or English, having been assigned male at birth, and identifying as feminine, including women, trans women, girls, ladyboys, shemales, transgender, and transsexual, or any other identity other than male/man/masculine. Recruitment, enrollment, and baseline data collection were accomplished from September to November 2016.

Participants were asked to attend a weekly 3-h transfacilitated group-delivered intervention in Beirut in February and March, 2017. Weekly meetings were held at a carefully chosen local NGO. The NGO was selected for its previous engagement with the community and because it is located in an area of Beirut where there are no major police/state security checkpoints nearby, thus improving the likelihood of participant safety. Follow-up data collection concluded in August, 2017. Institutional Review Boards at the University of California, San Francisco and the Lebanese American University reviewed and approved this study.

\section{Intervention and participation}

Baynetna retains the core components of TransAction: six to seven trans-facilitated group-delivered sessions, one-onone assessments, and a shared hot meal. The adapted intervention is intended to impact HIV prevention, as well as overall mental and sexual health, through facilitating improvements in gender affirmation satisfaction, community connectedness, and social cohesion. Through qualitative inquiry before pilot implementation, adaptations to TransAction were made for feasibility and cultural relevance.

Some examples of changes that were made include: language, such as the names of trans women in exemplar scenarios to names that are more common in Lebanon (e.g., Nadiya and Noor rather than Jane and Maria); available and accessible gender affirmation/transition processes relevant to the context (e.g., changing one's name and sex marker on government identification is very different in Lebanon vs. the United States); and context-specific strategies for harm reduction and violence reporting.

A facilitator, who is a trans woman, was trained to lead two groups of weekly discussions with a unique topical focus each week. Baynetna's six modules focus on: Gender Affirmation (week 1); self-esteem and transphobia (week 2); safer sex work, safer dating, and pleasure (week 3); HIV and other sexually transmitted infections (STIs) (week 4); family acceptance/rejection and violence (week 5); and working and skill building (week 6). Participants were able to receive a total of $\$ 100$ if they attended all study activities (enrollment; week 1 ; week 2; week 3; week 4; week 5; week 6; 1 month post-test; 3 months post-test; and 6 months post-test). Participants were also offered a list of local trans-friendly providers, condoms, and personal lubricant. At the end of the intervention, a festive celebration was held, during which participants shared a meal and cake and received certificates of completion.

\section{Data collection}

Baseline and follow-up questionnaire data were collected by a research team member using a combination of pen and paper and laptop computer using REDCap ${ }^{32}$ (hosted at University of California, San Francisco [UCSF]), a software program that facilitates quantitative data collection. Baseline data were collected at enrollment. Follow-up data were collected three times at 1,3 , and 6 months postintervention. The following description of measures reflect all the instruments that were used for data collection. In this study, we present results and relevant associations of hypothesized mechanisms of action in the following overall domains: gender affirmation; social support (from within trans communities); and mental and sexual health.

\section{Measures}

The following measures are a complete list of the scales and instruments used in this study. Selected measures for this analysis focus on the abovementioned overall domains and include: demographics; ever having had an STI; gender affirmation satisfaction; community connectedness (with other trans women); social cohesion (with other trans women); anxiety; depression; suicidality; and war event exposure. Measures are detailed below with those in this analysis noted in bold.

\section{Demographics}

Participants were asked questions at the beginning of the study about their demographics. Demographic information included: age; country of birth; refugee status; highest level of education; current average monthly income; financial status; importance of religion; relationship status; stability of living situation; current health insurance; current work; past and current sex work; and HIV status.

\section{Sexual health}

HIV and other STI testing was assessed using an 11-item instrument designed to capture: STI testing history, treatment, and likelihood for future testing; reasons for or against testing; HIV status; and engagement in care if HIV positive. Sexual risk behavior was measured through 13 items assessing number of partners, gender of partners, types of sex (insertive anal/receptive anal/insertive vaginal/receptive vaginal/transactional), and condom use. Riskiness of sexual encounters was measured through an adapted version of the 27 -item sexual situations instrument. ${ }^{33}$ Power dynamics in individuals' primary or most recent important relationship were measured using the 23-item Sexual Relationship Power Scale. ${ }^{34}$ Participants were also asked about engagement in sex work (past, current).

\section{Mental health}

Three screens for mental health symptoms were administered in addition to questions about suicidal behavior and three instruments to assess factors relevant to mental health. We screened for anxiety through seven anxiety-specific items in the Hospital Anxiety and Depression Scale (HADS), ${ }^{35}$ which has a range of possible scores from 0 to 21 with scores categorized as follows: normal 0-7; mild 8-10; moderate 11-14; and severe 15-21. Depression was screened for through the 9-item PHQ-9, ${ }^{36}$ with potential scores ranging from 0 to 27 and scores categorized as follows: minimal 0-4; mild 5-9; moderate 10-14; moderately severe 15-19; severe 20-27. 
Post-traumatic stress disorder (PTSD) was assessed using the 4-item Primary Care PTSD Screen, ${ }^{37}$ which allows for yes/no responses and yields a "positive" assessment for PTSD if a participant answers "yes" to any three items.

Participants were asked six questions about suicidality: suicidal thoughts (ever; past 3 months), plans (ever; past 3 months), and attempts (ever; past 3 months) with possible responses of yes, no, or decline to answer. We also measured participants' levels of acceptance from families of origin through an adapted instrument, family acceptance. ${ }^{38}$ Our adapted version contains nine items that assess trans-specific family acceptance/rejection. A history of lifetime trauma was measured through the 25-item Trauma History Questionnaire ${ }^{39}$ and a history of childhood trauma was captured through the 10-item adverse childhood experiences. ${ }^{40}$

\section{Social support}

Participants' social support was measured through three scales: (1) 13-item social cohesion, ${ }^{23}$ adapted for use among trans women (SC); (2) Gender Minority Stress and Resilience Measure $^{41}$ to assess community connectedness through 5 items (CC); and (3) the 12-item Multidimensional Scale of Perceived Social Support. ${ }^{42}$

\section{Gender affirmation, identity, and expression}

To measure some facets of participants' gender affirmation, identity, and expression, we asked questions about transgender group identity, gender typicality, “outness," physical/medical transition, and gender affirmation. An adapted 13-item transspecific version ${ }^{43}$ of the original Multigroup Ethnic Identity Measure $^{44}$ was administered to capture the extent to which participants identified with, associated with, and felt belonging to the local trans community. A six-item scale was adapted from the original gender typicality ${ }^{45}$ scale to determine how similar to or different from other trans women participants reported feeling; gender typicality has been associated with self-esteem among non-trans individuals.

In addition to questions about how participants think others view them, we administered an 11-item adapted trans-specific version of the Outness Inventory ${ }^{46}$ to determine the extent to which people in the participants' lives are aware of their trans identity. Thirty-one questions were used to capture participants' access, past, current, future desire for, and satisfaction with physical or medical transitionrelated changes (including hormone use, surgeries, and use of silicone and other injectable substances). Gender affirmation $^{22}$ was measured through a 22 -item scale; 5 items related to gender affirmation satisfaction were used for this analysis.

\section{Context}

Relevant contextual factors, including war event exposure and transphobia were assessed. War event exposure was measured through the full War Event Questionnaire, ${ }^{47}$ which was developed for and in Lebanon; 11 items were used for this analysis. Transphobia was assessed using the 35-item experiences of transphobia ${ }^{48}$ instrument, which was adapted from a scale measuring homophobia in gay and bisexual men. ${ }^{49}$

\section{Data analysis}

Summary scores were generated for anxiety (HADS), depression (PHQ-9), PTSD (Primary Care PTSD Screen), social cohesion (SC), community connectedness (CC), and war event exposure; change scores were calculated at each follow-up time point from baseline (1, 3, and 6 months postintervention). To assess the impact of participation in Baynetna on sexual health, mental health, and social support in this pilot study, we conducted the following statistical analyses.

Descriptive statistics were used to report demographics and proportional changes in gender affirmation satisfaction, community connectedness, and social cohesion. Each participant acted as her own control and we used paired $t$-tests to assess changes at follow-up from baseline to evaluate potential differences for outcomes measured on a continuous scale and McNemar's test for dichotomous outcomes. Spearman correlations were reported to assess associations between gender affirmation satisfaction and ever testing positive for an STI (other than HIV). Two-sided $p$-values $<.05$ were considered statistically significant. SAS v. 9.4 was used to perform the analysis.

Table 1. Participant Demographic Characteristics at Baseline $(N=16)$

\begin{tabular}{|c|c|}
\hline Variable & $\mathrm{n}(\%)$ \\
\hline \multicolumn{2}{|l|}{ Country of birth } \\
\hline Lebanon & $13(81)$ \\
\hline Iraq & $2(13)$ \\
\hline Syria & $1(6)$ \\
\hline \multicolumn{2}{|l|}{ Refugee status } \\
\hline Not a refugee & $13(81)$ \\
\hline Registered refugee & $3(19)$ \\
\hline \multicolumn{2}{|l|}{ Highest level of education } \\
\hline Completed primary school & $2(13)$ \\
\hline Did not complete secondary school & $2(13)$ \\
\hline Completed secondary school & $4(25)$ \\
\hline Some college/university & $6(38)$ \\
\hline Graduated from college/university & $2(13)$ \\
\hline \multicolumn{2}{|l|}{ Current average monthly income (from any source) } \\
\hline$\$ 0-500$ & $9(56)$ \\
\hline$\$ 501-1,000$ & $4(25)$ \\
\hline$\$ 1,001-2,000$ & $1(6)$ \\
\hline$\$ 2,001-3,000$ & $1(6)$ \\
\hline$\$ 3,001-4,000$ & $1(6)$ \\
\hline \multicolumn{2}{|l|}{ Financial status } \\
\hline I have enough money to live comfortably. & $3(19)$ \\
\hline I can barely manage with the money I have. & $7(44)$ \\
\hline I cannot manage with the money I have. & $6(38)$ \\
\hline Dichotomous importance of religion (yes) & $9(56)$ \\
\hline \multicolumn{2}{|l|}{ Relationship status } \\
\hline Unmarried and no partner & $8(50)$ \\
\hline Unmarried and serious partner & $5(31)$ \\
\hline Unmarried and casual partner(s) & $1(6)$ \\
\hline Married & $2(13)$ \\
\hline Dichotomous stability of living situation (yes) & $6(38)$ \\
\hline Dichotomous current health insurance (yes) & $4(25)$ \\
\hline Dichotomous currently working (yes) & $9(56)$ \\
\hline Dichotomous past sex work (yes) & $1(6)$ \\
\hline Dichotomous current sex work (yes) & $5(31)$ \\
\hline HIV status (positive) & $1(6)$ \\
\hline
\end{tabular}




\section{Results}

\section{Demographics}

Sixteen participants were enrolled at baseline and included in this analysis. The median age of the sample was 26 years, with a range from 22 to 50 years. Most participants were born in Lebanon (81\%); one participant was born in Syria and two participants were born in Iraq. All three participants who were not born in Lebanon were registered refugees. Seventyfive percent of the sample had completed secondary school whereas half had not studied at the college level. Most (81\%) said that they could not manage or could barely manage with the money/income they had. About half $(56 \%)$ of the sample said that religion is important to them. Half of the participants were unmarried without a serious partner. More than half $(63 \%)$ of the participants had unstable housing and most $(75 \%)$ were uninsured. Complete demographic data are summarized in Table 1.

\section{Intervention impact}

Although not powered for significance, the Baynetna pilot yielded promising positive trends of impact. Comparing those participants whose scores improved from baseline to 6month post-test to those participants whose scores did not improve demonstrated positive directionality with regard to intervention impact. Specifically, at the 6-month post-test, 7 out of 13 participants had improved gender affirmation satisfaction scores compared with baseline; 9 out of 13 participants had improved community connectedness scores compared with baseline; and 9 out of 13 participants had improved social cohesion scores. This relative increase in gender affirmation satisfaction, community connectedness, and social cohesion suggests a positive impact trend of the intervention, as shown in Table 2.
The pilot demonstrated an increase in proportion of participants testing for HIV between the 6 months before enrollment and the 6 months after enrollment. Specifically, 7 out of 15 participants $(47 \%)$ who reported being negative or unknown status at baseline had been tested for HIV during the 6 months before Baynetna enrollment (May-October 2016), whereas 11 out of 15 participants $(73 \%)$ reported HIV testing in the 6 months following enrollment (November 2016-April 2017).

\section{Hypothesized mechanisms of action}

Given the sample size, we did not expect to find statistically significant changes over time as the result of participation in the intervention, as described above. However, promising directionality of hypothesized mechanisms of action was detected with some statistical significance $(p=.05)$. These statistically significant results are described below and provided in Tables 3-5.

\section{Social support and mental health}

Results indicated a significant association between social support from other trans women and mental health. Specifically, higher social cohesion was significantly correlated with less suicidal thoughts at 3 months post-test $(p=.05)$. Additionally, there was a significant $(p=.019)$ relationship between more community connectedness and lower depression scores at 3 months post-test. Associations between social support and mental health are provided in Table 3.

\section{GA and STI history}

Because the Gender Affirmation model posits that when gender affirmation needs are met (i.e., gender affirmation satisfaction), HIV risk behavior is lower, we were interested in this hypothesized relationship. Furthermore, the Gender

Table 2. Baseline and 6-Month Post-test Gender Affirmation Satisfaction, Community Connectedness, and Social Cohesion Scores

\begin{tabular}{|c|c|c|c|c|c|c|}
\hline \multirow[b]{2}{*}{ Participant } & \multicolumn{2}{|c|}{$\begin{array}{l}\text { GAS score (GAS higher } \\
\text { score = more satisfaction })\end{array}$} & \multicolumn{2}{|c|}{$\begin{array}{c}C C(C C \text { higher score }=\text { more } \\
\text { connectedness })\end{array}$} & \multicolumn{2}{|c|}{$\begin{array}{c}S C(S C \text { lower score }=\text { more } \\
\text { cohesive })\end{array}$} \\
\hline & $\begin{array}{c}\text { Baseline } \\
(\mathrm{n}=16)\end{array}$ & $\begin{array}{l}\text { 6-month post-test } \\
\quad(\mathrm{n}=13)\end{array}$ & $\begin{array}{c}\text { Baseline } \\
(\mathrm{n}=16)\end{array}$ & $\begin{array}{l}\text { 6-month post-test } \\
(\mathrm{n}=13)\end{array}$ & $\begin{array}{c}\text { Baseline } \\
(\mathrm{n}=16)\end{array}$ & $\begin{array}{l}\text { 6-month post-test } \\
(\mathrm{n}=13)\end{array}$ \\
\hline 1 & 16 & 11 & 17 & 10 & 36 & 39 \\
\hline 2 & 22 & 27 & 9 & 13 & 49 & 34 \\
\hline 3 & 15 & 26 & 10 & 10 & 40 & 32 \\
\hline 4 & 20 & 25 & 11 & 3 & 43 & 41 \\
\hline 5 & 21 & 21 & 12 & 15 & 37 & 32 \\
\hline 6 & 24 & ND & 7 & 13 & 40 & ND \\
\hline 7 & 11 & ND & 17 & 17 & 31 & ND \\
\hline 8 & 23 & 21 & 12 & ND & 27 & 36 \\
\hline 9 & 22 & 21 & 16 & ND & 44 & 35 \\
\hline 10 & 23 & 16 & 6 & 7 & 45 & 37 \\
\hline 11 & 19 & 23 & 6 & 15 & 40 & 33 \\
\hline 12 & 17 & 17 & 14 & 15 & 27 & 27 \\
\hline 13 & 9 & 17 & 13 & 17 & 40 & 31 \\
\hline 14 & 13 & ND & 16 & ND & 41 & ND \\
\hline 15 & 16 & 23 & 12 & 14 & 31 & 34 \\
\hline 16 & 12 & 17 & 8 & 13 & 47 & 38 \\
\hline $\begin{array}{l}\text { Proportion of score } \\
\text { improvement }\end{array}$ & \multicolumn{2}{|r|}{$7 / 13$} & \multicolumn{2}{|r|}{$9 / 13$} & \multicolumn{2}{|r|}{$9 / 13$} \\
\hline
\end{tabular}

CC, community connectedness; GAS, gender affirmation satisfaction; ND, no data; SC, social cohesion. 
Table 3. Community Connectedness, Social Cohesion, and Mental Health (AnXiety, Depression, Post-Traumatic Stress Disorder, and Suicidal Thoughts) at Baseline, 1-Month Post-test, 3-Month Post-test, and 6-Month Post-test

Factors ( $\mathrm{n}=$ number of participants)

Average difference

Higher SC and higher anxiety $(n=16)^{\mathrm{a}}$

Higher SC and lower anxiety $(n=15)^{\mathrm{b}}$

Higher SC and lower anxiety $(n=13)^{\mathrm{c}}$

Higher SC and lower anxiety $(n=13)^{\mathrm{d}}$ (95\% CI of the difference)

\section{$\mathrm{p}$}

$1.0(-0.5$ to 0.5$)$

$-0.3(-0.7$ to 0.3$)$

$-0.5(-0.8$ to 0.1$)$

$-0.3(-0.7$ to 0.3$)$

p

Higher SC and lower depression scores ${ }^{\mathrm{a}}$

$$
\begin{aligned}
& 0-4(n=5) \\
& 5-9(n=3) \\
& 10-14(n=5) \\
& 15-19(n=1) \\
& 20-27(n=2)
\end{aligned}
$$

$11.0(8$ to 25$)$

12.0 (3 to 25$)$

12.0 (5 to 21$)$

16.0 (16 to 16$)$

9.5 (7 to 12$)$

Higher SC and lower depression scores ${ }^{\mathrm{b}}$

$0-4(n=8)$

5-9 $(n=3)$

$10-14(n=1)$

$15-19(n=2)$

20-27 $(n=1)$

$10.0(3$ to 25$)$

$12.0(12$ to 12$)$

$15.0(15$ to 15$)$

18.5 (12 to 25$)$

16.0 (16 to 16$)$

Higher SC and lower depression scores ${ }^{\mathrm{c}}$

$0-4(n=4)$

$5-9(n=5)$

$10-14(n=2)$

$15-19(n=2)$

20-27 $(n=0)$

$11.5(8$ to 25$)$

15.0 ( 3 to 25$)$

8.5 (5 to 12 )

12.5 (9 to 16 )

NA

Higher SC and lower depression scores ${ }^{\mathrm{d}}$

$0-4(n=5)$

5-9 $(n=4)$

10-14 $(n=2)$

$15-19(n=1)$

$20-27(n=1)$

Higher SC for those without PTSD $(n=10)$ than those with PTSD $(n=6)^{\mathrm{a}}$

Higher SC for those without PTSD $(n=10)$ than those with PTSD $(n=5)^{\mathrm{b}}$

Higher SC for those without PTSD $(n=7)$ than those with PTSD $(n=6)^{\mathrm{c}}$

Higher SC for those without PTSD $(n=10)$ than those with PTSD $(n=3)^{\mathrm{d}}$

Higher SC and less suicidal thoughts $(n=16)^{\mathrm{a}}$

Higher SC and less suicidal thoughts $(n=14)^{\mathrm{b}}$

Higher SC and less suicidal thoughts $(n=12)^{\mathrm{c}}$

Higher SC and less suicidal thoughts $(n=13)^{\mathrm{d}}$

\begin{tabular}{lcc}
\hline Factors & Spearman correlation (95\% CI) & $\mathrm{p}$ \\
\hline Higher CC and lower anxiety $(n=16)^{\mathrm{a}}$ & $-0.1(-0.5$ to 0.5$)$ & .84 \\
Higher CC and lower anxiety $(n=15)^{\mathrm{b}}$ & $-0.2(-0.7$ to 0.3$)$ & .40 \\
Higher CC and lower anxiety $(n=13)^{\mathrm{c}}$ & $0.1(-0.5$ to 0.6$)$ & .72 \\
Higher CC and lower anxiety $(n=13)^{\mathrm{d}}$ & $-0.2(-0.7$ to 0.4$)$ & .46 \\
\hline Factors & Median (minimum to maximum) & $\mathrm{p}$ \\
\hline CC and depression scores & & $.44^{\mathrm{f}}$ \\
$0-4(n=5)$ & & \\
$5-9(n=3)$ & $14(11$ to 16$)$ & \\
$10-14(n=2)$ & $9(7$ to 12$)$ & \\
$15-19(n=1)$ & $11(6$ to 16$)$ & \\
$20-27(n=2)$ & $8(6$ to 17$)$ & \\
\hline
\end{tabular}

$8.0(3$ to 21$)$

12.0 (9 to 25$)$

18.5 (12 to 25$)$

$15.0(15$ to 15$)$

7.0 (7 to 7$)$

$1.1(-6.5$ to 8.8$)$

$6.6(-0.6$ to 13.8$)$

$5.4(-2.8$ to 13.5$)$

$1.8(-8.9$ to 12.5$)$

-2.2 (-9.6 to 5.2$)$

$0.9(-7.4$ to 9.1$)$

$-7.8(-15.7$ to -0.0$)$

$-5.1(-13.8$ to 3.6$)$ 
TABle 3. (Continued)

\begin{tabular}{lc}
\hline Factors & Median (minimum to maximum) \\
\hline CC and lower depression scores & $\mathrm{p}$ \\
$0-4(n=8)$ & $13(8$ to 16$)$ \\
$5-9(n=3)$ & $7(6$ to 10$)$ \\
$10-14(n=1)$ & $12(12$ to 12$)$ \\
$15-19(n=2)$ & $12.5(12$ to 13$)$ \\
$20-27(n=1)$ & $17(17$ to 17$)$ \\
CC and lower depression scores ${ }^{\mathrm{c}}$ & \\
$0-4(n=4)$ & $14.5(13$ to 16$)$ \\
$5-9(n=5)$ & $12(9$ to 12$)$ \\
$20-27(n=0)$ & $\mathrm{NA}$ \\
Similar CC across depression scores ${ }^{\mathrm{d}}$ & $8.0(3$ to 21$)$ \\
$0-4(n=5)$ & $12(9$ to 25$)$ \\
$5-9(n=4)$ & $12(6$ to 12$)$ \\
$10-14(n=3)$ & NA \\
$15-19(n=0)$ & $6(6$ to 6$)$ \\
$20-27(n=1)$ & $.019^{*}$ \\
\hline
\end{tabular}

Factors

Higher CC and lower PTSD $(n=16)^{\mathrm{a}}$

Higher CC and lower PTSD $(n=15)^{\mathrm{b}}$

Higher CC and lower PTSD $(n=13)^{\mathrm{c}}$

Higher CC and lower PTSD $(n=13)^{\mathrm{d}}$

Higher CC and less suicidal thoughts $(n=16)^{\mathrm{a}}$

Higher CC and less suicidal thoughts $(n=14)^{\mathrm{b}}$

Higher CC and less suicidal thoughts $(n=12)^{\mathrm{c}}$

Higher CC and less suicidal thoughts $(n=13)^{\mathrm{d}}$

Average difference (95\% CI)

$\begin{array}{ll}-0.8(-4.9 \text { to } 3.3) & .68 \\ -1.1(-5.2 \text { to } 3.0) & .57 \\ -0.1(-4.2 \text { to } 3.9) & .94 \\ 1.9(-2.9 \text { to } 6.8) & .65 \\ 0.6(-3.4 \text { to } 4.6) & .74 \\ 2.4(-1.6 \text { to } 6.4) & .21 \\ 0.33(-4.1 \text { to } 4.8) & .87 \\ -1.1(-5.3 \text { to } 3.2) & .60\end{array}$

${ }^{\mathrm{a}}$ Measured at baseline.

${ }^{\mathrm{b}}$ Measured at 1-month follow-up.

${ }^{\mathrm{c}}$ Measured at 3-month follow-up.

${ }^{\mathrm{d}}$ Measured at 6-month follow-up.

${ }^{\mathrm{e}}$ Two-sided $p$-value generated from two-sample $t$-test.

${ }^{\mathrm{f}}$ Two-sided Kruskal-Wallis $p$-value calculated after combining 15-19 with 20-27 depression scores.

${ }^{\mathrm{g}}$ Two-sided Kruskal-Wallis $p$-value calculated after combining 10-14, 15-19 with 20-27 depression scores.

*Significant $(p \leq .05)$.

CI, confidence interval; NA, not applicable; NE, not estimable since only one participant reported; PTSD, post-traumatic stress disorder.

Affirmation model had not been tested outside the United States, so it was important to determine whether associations identified in the United States held true in a novel setting in the Global South, in this case, MENA. Therefore, we examined participants' histories of STIs in relationship to gender affirmation satisfaction. At baseline, those who reported never having had an STI had higher gender affirmation satisfaction scores (7.5, 95\% confidence interval: 1.4-13.6) than those who had ever had an STI $(p=.019)$. Table 4 provides the associations between gender affirmation satisfaction and STI history at baseline, 1 month post-test, 3 months post-test, and 6 months post-test.

Table 4. Gender Affirmation Satisfaction and Ever Having an STI Other than HiV at Baseline, 1-Month Post-test, 3-Month Post-test, and 6-Month Post-test

\begin{tabular}{lcc}
\hline Factors & Average difference $(95 \%$ CI $)$ & $\mathrm{p}$ \\
\hline Higher GAS for those without an STI $(n=14)$ than those with an STI $(n=2)^{\mathrm{a}}$ & $7.5(1.4$ to 13.6$)$ & $1.6(-3.6$ to 6.7$)$ \\
Higher GAS for those without an STI $(n=13)$ than those with an STI $(n=2)^{\mathrm{b}}$ & $-1.7(-8.3$ to 4.8$)$ & $.52^{\mathrm{e}}$ \\
Lower GAS for those without an STI than those with an STI $(n=13)^{\mathrm{c}}$ & $-57^{\mathrm{e}}$ \\
Lower GAS for those without an STI $(n=12)$ than those with an STI $(n=1)^{\mathrm{d}}$ & $-3.7(-13.9$ to 6.6) \\
\hline
\end{tabular}

"With an STI" and "without an STI" refer to the participant reporting having ever or having never had an STI other than HIV, respectively.

${ }^{\mathrm{a}}$ Measured at baseline.

${ }^{\mathrm{b}}$ Measured at 1-month follow-up.

${ }^{c}$ Measured at 3-month follow-up.

${ }^{\mathrm{d}}$ Measured at 6-month follow-up.

${ }^{\mathrm{e}}$ Two-sided $p$-value generated from two-sample $t$-test.

$*$ Significant $(p \leq .05)$.

STI, sexually transmitted infection. 
Table 5. War Event Exposure and Mental Health (AnXiety, Depression, Post-Traumatic Stress Disorder, and Suicidal Thoughts) at Baseline, 1-Month Post-test, 3-Month Post-test, and 6-Month Post-test

\begin{tabular}{|c|c|c|}
\hline Factors $(\mathrm{n}=$ number of participants $)$ & Spearman correlation (95\% CI) & $\mathrm{p}$ \\
\hline $\begin{array}{l}\text { Higher WEQ correlated with lower anxiety }(n=16)^{\mathrm{a}} \\
\text { Higher WEQ correlated with lower anxiety }(n=15)^{\mathrm{b}} \\
\text { Higher WEQ correlated with lower anxiety }(n=13)^{\mathrm{c}} \\
\text { Higher WEQ correlated with lower anxiety }(n=13)^{\mathrm{d}}\end{array}$ & $\begin{array}{l}-0.6(-0.8 \text { to }-0.1) \\
-0.7(-0.9 \text { to }-0.3) \\
-0.4(-0.8 \text { to } 0.2) \\
-0.5(-0.8 \text { to } 0.1)\end{array}$ & $\begin{array}{l}.02 * \\
.004 * \\
.19 \\
.08\end{array}$ \\
\hline Factors & Median (minimum to maximum) & $\mathrm{p}$ \\
\hline $\begin{array}{l}\text { WEQ with depression scores } \\
\text { a-4 } \\
\text { 5-9 }(n=5) \\
10-14(n=5) \\
15-19(n=1) \\
20-27(n=2)\end{array}$ & $\begin{array}{l}3(1 \text { to } 6) \\
2(1 \text { to } 4) \\
6(4 \text { to } 8) \\
4(4 \text { to } 4) \\
4(1 \text { to } 7)\end{array}$ & $.08^{\mathrm{f}}$ \\
\hline $\begin{array}{l}\text { WEQ with depression scores }{ }^{\mathrm{b}} \\
0-4(n=8) \\
5-9(n=3) \\
10-14(n=1) \\
15-19(n=2) \\
20-27(n=1)\end{array}$ & $\begin{array}{l}3(1 \text { to } 7) \\
4(1 \text { to } 6) \\
6(6 \text { to } 6) \\
5(2 \text { to } 8) \\
4(4 \text { to } 4)\end{array}$ & $.47^{\mathrm{g}}$ \\
\hline $\begin{array}{l}\text { WEQ with depression scores } \\
\text { 0-4 } \\
\text { 5-9 }(n=4) \\
10-14(n=2) \\
15-19(n=2) \\
\text { 20-27 }(n=0)\end{array}$ & $\begin{array}{l}4.5(1 \text { to } 8) \\
1.0(1 \text { to } 6) \\
6.5(6 \text { to } 7) \\
3.5(3 \text { to } 4) \\
\text { NA }\end{array}$ & $.58^{\mathrm{g}}$ \\
\hline $\begin{array}{l}\text { WEQ with depression scores } \\
\text { d } \\
\text { 0-4 }(n=5) \\
\text { 5-9 }(n=4) \\
10-14(n=2) \\
15-19(n=1) \\
20-27(n=1)\end{array}$ & $\begin{array}{l}4(1 \text { to } 7) \\
2(1 \text { to } 8) \\
4(2 \text { to } 6) \\
6(6 \text { to } 6) \\
7(7 \text { to } 7)\end{array}$ & $.58^{\mathrm{g}}$ \\
\hline
\end{tabular}

Factors
WEQ with PTSD $(n=16)^{\mathrm{a}}$
WEQ with PTSD $(n=15)^{\mathrm{b}}$
WEQ with PTSD $(n=13)^{\mathrm{c}}$
WEQ with PTSD $(n=13)^{\mathrm{d}}$

Average difference $(95 \%$ CI)

$\mathrm{p}$

\begin{tabular}{lcc}
\hline Factors & Average difference $(95 \%$ CI $)$ & $\mathrm{p}$ \\
\hline $\begin{array}{l}\text { Lower WEQ for those with suicidal thoughts in the last } 3 \text { months } \\
(n=3) \text { than those without }(n=13)^{\mathrm{a}}\end{array}$ & $-2.5(-5.6$ to 1.0$)$ \\
$\begin{array}{l}\text { Higher WEQ for those with suicidal thoughts in the last } 3 \text { months } \\
(n=1) \text { than those without }\left(n=14^{\mathrm{DK}}\right)^{\mathrm{b}}\end{array}$ & $4.5(-0.3$ to 9.3$)$ \\
$\begin{array}{l}\text { Higher WEQ for those with suicidal thoughts in the last } 3 \text { months } \\
(n=1) \text { than those without }\left(n=12^{\mathrm{DK}}\right)^{\mathrm{c}}\end{array}$ & $3.5(-2.2$ to 9.2$)$ \\
$\begin{array}{l}\text { Similar WEQ for those with suicidal thoughts in the last } 3 \\
\text { months }(n=3) \text { than those without }(n=10)^{\mathrm{d}}\end{array}$ & $-0.1(-4.2$ to 4.0$)$
\end{tabular}

${ }^{\mathrm{a}}$ Measured at baseline.

${ }^{\mathrm{b}}$ Measured at 1-month follow-up.

${ }^{\mathrm{c}}$ Measured at 3-month follow-up.

${ }^{\mathrm{d}}$ Measured at 6-month follow-up.

${ }^{\mathrm{e}}$ Two-sided $p$-value generated from two-sample $t$-test.

${ }^{\mathrm{f}}$ Two-sided Kruskal-Wallis $p$-value calculated after combining 15-19 with 20-27 depression scores.

${ }^{\mathrm{g}}$ Two-sided Kruskal-Wallis $p$-value calculated after combining 10-14, 15-19 with 20-27 depression scores.

*Significant $(p \leq .05)$.

DK, “don't know" responses $(n=1)$ included in those without an STI; WEQ, score on War Event Questionnaire measuring war event exposure. 


\section{War event exposure and mental health}

Given the context of protracted political violence in Lebanon, it was important to assess war exposure's impact on participant health. As anticipated, more war event exposure was associated with higher anxiety at baseline $(p=.02)$ and at 1 month post-test $(p=.004)$. Table 5 provides war event exposure and its associations with all mental health conditions assessed at each timepoint.

\section{Integrated theoretical model}

The outcomes of this study also contributed to development of an integrated theoretical model, as follows. The basis of the integrated theoretical model draws from existing literature that illustrates relevant factors that facilitate and impede access to and need for gender affirmation. The intended outcomes include decreases in mental health symptoms and increases in HIV prevention and treatment engagement. The integrated theoretical model incorporates the results from this study and the previous study in Lebanon upon which this study was built. The goal of Baynetna is to improve sexual and mental health outcomes by intervening on community connectedness, social cohesion, and gender affirmation needs. The visual integrated conceptual model is provided in Figure 1.

\section{Discussion}

Although this pilot was not designed or powered to detect significant associations, the results elucidate some promising trends that contribute to the existing body of literature and inform global efforts to improve the health and therefore quality of life among trans women. Demographic characteristics, despite the use of convenience sampling, represent a wide range of diversity, including age and refugee status. We note that there were no Palestinian refugee participants; this may represent a limitation, which will be addressed in future studies to ensure that we are capturing the full range of identities, backgrounds, and cultural groups within trans communities in Lebanon. We were not surprised that a staggering $81 \%$ of the sample reported that they could not or could barely manage financially. Financial instability as a common challenge has been well documented in the existing research, ${ }^{50,51}$ which often leads to sexual, mental, and physical risk. ${ }^{6}$

Increases in the proportions of participants whose community connectedness, social cohesion, and gender affirmation satisfaction scores improved post-intervention confirm the proposed mechanisms of impact of Baynetna. Additionally, the increase in proportion of participants testing for HIV between the 6 months before enrollment and the 6 months after enrollment supports evidence for successful achievement of intervention objectives.

The statistically significant results further contribute to our confidence in Baynetna's hypothesized mechanisms of action. That higher social cohesion was associated with less suicidal thoughts suggests that bolstering social cohesion can positively impact mental health-specifically suicide ideationamong trans women in Lebanon. Likewise, the significant correlation between community connectedness and lower depression scores indicates this positive impact that community connectedness has on mental health-in this case, depression symptoms. Given the high rates of suicide ideation and attempts within the community, especially compared with

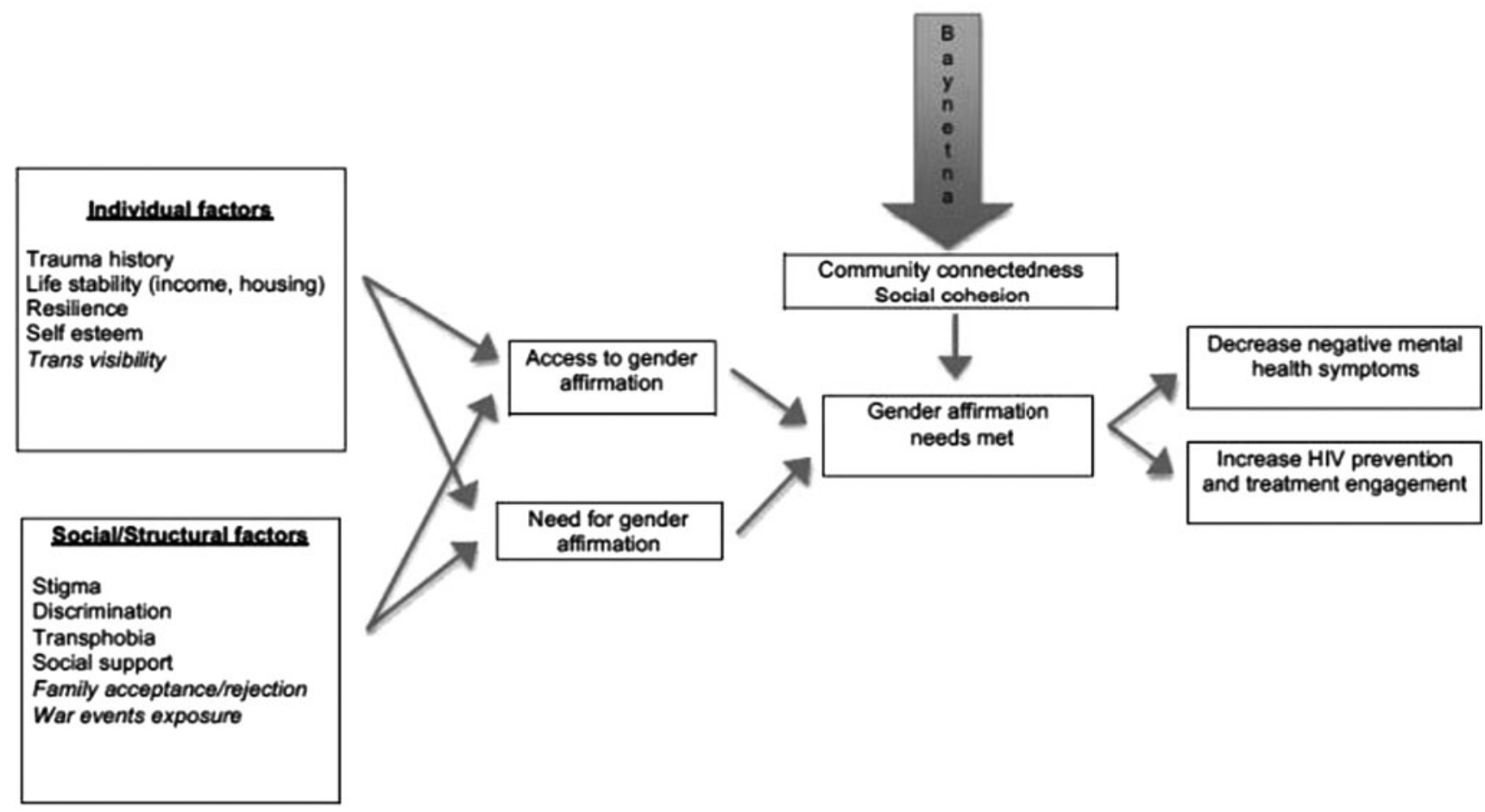

FIG. 1. Integrated conceptual framework: Baynetna. The following italicized factors have been added based on results and findings in Lebanon: trans visibility or "outness", ${ }^{20}$; family acceptance/rejection"; and war event exposure (present article). Nonitalicized components of gender affirmation ${ }^{22}$, social cohesion, ${ }^{23}$ and community connectedness, ${ }^{41}$ are based on existing research. 
the $2 \%$ rate in Lebanon's general population, trans-informed suicide prevention and mental health treatment are essential, particularly in the context of systemic and structural marginalization.

These pilot results also support the idea that the Gender Affirmation model is applicable to the Lebanese context; that greater gender affirmation satisfaction scores were associated with having never had an STI aligns with the hypothesized relationship between risk for STI infection and gender affirmation satisfaction.

The research team agreed that it was important to assess the trauma that trans women have experienced and that Lebanon/ region-specific trauma should include exposure to war events. Not surprisingly, we found a significant association between war event exposure and higher anxiety. This result highlights the importance of trauma-informed and context-specific intervention approaches in addition to the challenges of measuring HIV prevalence in the context of regional conflict and displaced individuals. ${ }^{52}$

In summary, these significant results suggest that bolstering social cohesion and community connectedness can positively impact mental health-specifically depression and suicidal thoughts. The trend in gender affirmation satisfaction supports the anticipated relationship between gender affirmation satisfaction and less risk for STI infection. Finally, as hypothesized, higher anxiety and war event exposure are highly correlated, indicating the need for trauma-informed intervention modalities.

\section{Conclusion}

We have shown for the first time that our adapted intervention, Baynetna, and the constructs upon which it is builtsocial cohesion, community connectedness, and gender affirmation -is applicable and has positive impact trends in this novel context outside the United States. Taken together, these results support the hypothesis that gender affirmation satisfaction, community connectedness, and social cohesion from within communities of trans women in Lebanon are important constructs for understanding and mitigating sexual and mental health risk behavior.

A context of protracted war exposure negatively impacts overall health in this population and must be attended to in all culturally responsive strategies for health promotion. Future research will determine larger-scale feasibility and acceptability in a randomized controlled trial to compare the effects in those who received the intervention versus those who did not. Participants will be followed for a longer period of time, such as 12 months, to evaluate longer-term retention of hypothesized intervention effects. If found to be effective, efforts to scale up would leverage long-term relationships with LGBTQ+ leaders, community, and organizations for intervention integration into existing infrastructure. Future directions also include adaptation to and testing in other countries impacted by war exposure both within and outside MENA.

\section{Acknowledgments}

First and foremost, the authors wish to acknowledge and deeply thank their participants in the study, for showing up, engaging, trusting, and sharing with them and one another. Additionally, the hard work, dedication, and commitment of each research team member was essential for the success of this pilot study, for which they are truly grateful. They would also like to acknowledge Dr. Maya Sen for early contributions to analytic guidance.

\section{Disclaimer}

The content is solely the responsibility of the authors and does not necessarily represent the official views of the $\mathrm{Na}$ tional Institutes of Health.

\section{Author Disclosure Statement}

No competing financial interests exist.

\section{Funding Information}

Research reported in this publication was supported by the National Institutes of Mental Health of the National Institutes of Health, under award number K01MH102142. Additionally, this publication's statistical assistance was supported by the National Center for Advancing Translational Sciences, National Institutes of Health, through UCSF-CTSI UL1 TR001872.

\section{References}

1. Kaplan RL, Sevelius J, Ribeiro K: In the name of brevity: The problem with binary HIV risk categories. Glob Public Health 2016;11:824-834.

2. Baral SD, Poteat T, Stromdahl S, Wirtz AL, Guadamuz TE, Beyrer C: Worldwide burden of HIV in transgender women: A systematic review and meta-analysis. Lancet Infect Dis 2013;13:214-222.

3. Wirtz AL, Clouse E, Veronese V, et al.: New HIV testing technologies in the context of a concentrated epidemic and evolving HIV prevention: Qualitative research on HIV selftesting among men who have sex with men and transgender women in Yangon, Myanmar. J Int AIDS Soc 2017;20:21796.

4. Pitasi MA, Oraka E, Clark H, Town M, DiNenno EA: HIV testing among transgender women and men-27 States and Guam, 2014-2015. Morb Mortal Wkly Rep 2017;66:883-887.

5. Hines DD, Draucker CB, Habermann B: HIV testing and entry to care among trans women in Indiana. J Assoc Nurses AIDS Care 2017;28:723-736.

6. Kaplan RL, Wagner GJ, Nehme S, Aunon F, Khouri D, Mokhbat J: Forms of safety and their impact on health: An exploration of HIV/AIDS-related risk and resilience among trans women in Lebanon. Health Care Women Int 2015;36: 917-935.

7. Gökengin D, Doroudi F, Tohme J, Collins B, Madani N: HIV/AIDS: Trends in the Middle East and North Africa region. Int J Infect Dis 2016;44:66-73.

8. (UNAIDS) JUNPoHA: 90-90-90: An ambitions treatment target to help end the AIDS epidemic, 2014. www.unaids .org/sites/default/files/media_asset/90-90-90_en_0.pdf accessed August 10, 2019.

9. UNAIDS: UNAIDS Country Report: Lebanon, 2016. www .unaids.org/en/regionscountries/countries/lebanon accessed August 10, 2019.

10. Kaplan RL, McGowan J, Wagner GJ: HIV prevalence and demographic determinants of condomless receptive anal intercourse among trans feminine individuals in Beirut, Lebanon. J Int AIDS Soc 2016;19(3 Suppl. 2):20787.

11. Kaplan RL, Nehme S, Aunon F, de Vries D, Wagner G: Suicide risk factors among trans feminine individuals in Lebanon. Int J Transgender 2016;17:23-30. 
12. Wagner GJ, Tohme J, Hoover M, et al:: HIV prevalence and demographic determinants of unprotected anal sex and HIV testing among men who have sex with men in Beirut, Lebanon. Arch Sex Behav 2014;43:779-788.

13. Mahfoud Z, Afifi R, Ramia S, et al:: HIV/AIDS among female sex workers, injecting drug users and men who have sex with men in Lebanon: Results of the first biobehavioral surveys. AIDS (London, England) 2010;24(Suppl. 2):S45S54.

14. Budge SL, Adelson JL, Howard KL: Anxiety and depression in transgender individuals: The roles of transition status, loss, social support, and coping. J Consult Clin Psychol 2013;81:545-557.

15. Clements-Nolle K, Marx R, Katz M: Attempted suicide among transgender persons: The influence of gender-based discrimination and victimization. J Homosex 2006;51: 53-69.

16. Gonzalez CA, Bockting WO, Beckman LJ, Durán RE: Agentic and communal personality traits: Their association with depression and resilience among transgender women. Sex Roles 2012;67:528-544.

17. Clements-Nolle K, Marx R, Katz M: HIV prevalence, risk behaviors, health care use, and mental health status of transgender persons: Implications for public health intervention. Am J Public Health 2001;91:915-921.

18. Herbst JH, Jacobs ED, Finlayson TJ, McKleroy VS, Neumann MS, Crepaz N; HIV/AIDS Prevention Research Synthesis Team: Estimating HIV prevalence and risk behaviors of transgender persons in the United States: A systematic review. AIDS Behav 2008;12:1-17.

19. Weissman MM, Bland RC, Canino GJ, et al.: Prevalence of suicide ideation and suicide attempts in nine countries. Psychol Med 1999;29:9-17.

20. Reback CJ, Clark K, Fletcher JB: TransAction: A homegrown, theory-based, HIV risk reduction intervention for transgender women experiencing multiple health disparities. Sex Res Soc Policy 2018;16:408-418.

21. Wingood GM, DiClemente RJ: The ADAPT-ITT model: A novel method of adapting evidence-based HIV interventions. J Acquir Immune Defic Syndr (1999) 2008;47(Suppl. 1):S40-S46.

22. Sevelius JM: Gender affirmation: A framework for conceptualizing risk behavior among transgender women of color. Sex Roles 2013;68:675-689.

23. Lippman SA, Donini A, Diaz J, Chinaglia M, Reingold A, Kerrigan D: Social-environmental factors and protective sexual behavior among sex workers: The Encontros intervention in Brazil. Am J Public Health 2010;100(Suppl. 1): S216-S223.

24. Meyer IH: Prejudice, social stress, and mental health in lesbian, gay, and bisexual populations: Conceptual issues and research evidence. Psychol Bull 2003;129:674-697.

25. Frost DM, Meyer IH: Measuring community connectedness among diverse sexual minority populations. J Sex Res 2012;49:36-49.

26. Austin A, Goodman R: The impact of social connectedness and internalized transphobic stigma on self-esteem among transgender and gender non-conforming adults. J Homosex 2017;64:825-841.

27. Sanchez FJ, Vilain E: Collective self-esteem as a coping resource for male-to-female transsexuals. J Couns Psychol 2009;56:202-209.

28. Bariola E, Lyons A, Leonard W, Pitts M, Badcock P, Couch M: Demographic and psychosocial factors associated with psychological distress and resilience among transgender individuals. Am J Public Health 2015;105:2108-2116.

29. Stanton MC, Ali S, Chaudhuri S: Individual, social and community-level predictors of wellbeing in a US sample of transgender and gender non-conforming individuals. Cult Health Sex 2017;19:32-49.

30. Testa RJ, Jimenez CL, Rankin S: Risk and resilience during transgender identity development: The effects of awareness and engagement with other transgender people on affect. J Gay Lesbian Ment Health 2014;18:31-46.

31. Kaplan RL, El Khoury C, Lize N, Wehbe S, Mokhbat J: Feasiblity and acceptablity of a behavioral group support invervention among transgender women: A sexual and mental health mixed methods pilot study in Beirut, Lebanon. AIDS Educ Prev 2019;31:246-258.

32. Harris PA, Taylor R, Thielke R, Payne J, Gonzalez N, Jose CG: Research electronic data capture (REDCap)A metadata-driven methodology and workflow process for providing translational research informatics support. J Biomed Inform 2009;42:377-381.

33. Diaz R, Ayala G, Bein E: Sexual risks as an outcome of social oppression: Data from a probablity sample of Latino gay men in three US cities. Cultur Divers Ethnic Minor Psychol 2004;10:255-267.

34. Pulerwitz J, Gortmaker SL, DeJong W: Measuring relationship power in HIV/STD research. Sex Roles 2000;42: 637-660.

35. Zigmond AS, Snaith RP: The hospital anxiety and depression scale. Acta Psychiatr Scand 1983;67:361-370.

36. Kroenke K, Spitzer RL, Williams JB: The PHQ-9: Validity of a brief depression severity measure. J Gen Intern Med 2001;16:606-613.

37. Boscarino JA, Kirchner HL, Hoffman SN, Sartorius J, Adams RE, Figley CR: A brief screening tool for assessing psychological trauma in clinical practice: Development and validation of the New York PTSD Risk Score. Gen Hosp Psychiatry 2011;33:489-500.

38. Grant JM, Mottet LA, Tanis J, Harrison J, Herman J, Keisling M: Injustice at Every Turn: A Report of the National Transgender Discrimination Survey. Washington, DC: National Center for Transgender Equality and National Gay and Lesbian Task Force, 2011.

39. Hooper LM, Stockton P, Krupnick JL, Green BL: Development, use, and psychometric properties of the trauma history questionnaire. J Loss Trauma 2011;16: 258-283.

40. Felitti VJ, Anda RF, Nordenberg D, et al.: Relationship of childhood abuse and household dysfunction to many of the leading causes of death in adults: The Adverse Childhood Experiences (ACE) Study. Am J Prev Med 1998;14:245258.

41. Testa RJ, Habarth J, Peta J, Balsam K, Bockting W: Development of the gender minority stress and resilience measure. Psychol Sex Orient Gender Divers 2015;2: 65-77.

42. Zimet GD, Dahlem NW, Zimet SG, Farley GK: The multidimensional scale of perceived social support. J Pers Assess 1988;52:30-41.

43. Jefferson K, Neilands TB, Sevelius J: Transgender women of color: Discrimination and depression symptoms. Ethn Inequal Health Soc Care 2013;6:121-136.

44. Phinney J: The multigroup ethnic identity measure: A new scale for use with diverse groups. J Adolesc Res 1992;7: 156-176. 
45. Tate CC, Bettergarcia JN, Brent LM: Re-assessing the role of gender-related cognitions for self-esteem: The importance of gender typicality for cisgender adults. Sex Roles 2015;72:221.

46. Mohr JJ, Fassinger RE: Measuring dimensions of lesbian and gay male experience. Meas Eval Couns Dev 2000;33: 66-90.

47. Karam EG, al-Atrash R, Saliba S, Melhem N, Howard D: The war events questionnaire. Soc Psychiatry Psychiatr Epidemiol 1999;34:265-274.

48. Sugano E, Nemoto T, Operario D: The impact of exposure to transphobia on HIV risk behavior in a sample of transgendered women of color in San Francisco. AIDS Behav 2005;10:217.

49. Diaz RM, Ayala G, Bein E, Henne J, Marin BV: The impact of homophobia, poverty, and racism on the mental health of gay and bisexual Latino men: Findings from 3 US cities. Am J Public Health 2001;91:927-932.

50. Logie $\mathrm{CH}$, Abramovich A, Schott N, Levermore K, Jones $\mathrm{N}$ : Navigating stigma, survival, and sex in contexts of so- cial inequity among young transgender women and sexually diverse men in Kingston, Jamaica. Reprod Health Matters 2018:1-12.

51. Crissman HP, Berger MB, Graham LF, Dalton VK: Transgender demographics: A household probability sample of US adults, 2014. Am J Public Health 2017;107:213-215.

52. Zea MC, Reisen CA, Bianchi FT, et al.: Armed conflict, homonegativity and forced internal displacement: Implications for HIV among Colombian gay, bisexual and transgender individuals. Cult Health Sex 2013;15:788-803.

Address correspondence to: Rachel L. Kaplan

Obstetrics, Gynecology, and Reproductive Sciences University of California, San Francisco (UCSF) 550 16th Street San Francisco, CA 94158 USA

E-mail: rachel.kaplan@ucsf.edu 